\title{
AUTOMORPHISM INVARIANT CARTAN SUBGROUPS AND POWER MAPS OF DISCONNECTED GROUPS
}

\author{
PRALAY CHATTERJEE
}

\begin{abstract}
We extend a well-known result of R. Steinberg on the existence of an invariant maximal torus under a semisimple automorphism of an algebraic group over an algebraically closed field. We show that the same result holds when the underlying field is of characteristic zero, but not necessarily algebraically closed. We next study surjectivity of the power maps $g \mapsto g^{n}$ of disconnected algebraic groups of characteristic zero. In the case of disconnected real algebraic groups we apply our generalisation of Steinberg's result to obtain results on the surjectivity of the power maps. We also extend a result of A. Borel on weak exponentiality in real Lie groups by relating it with the surjectivity of the square map.
\end{abstract}

\section{IntRoduCtion AND THE MAIN RESUlts}

The purpose of this paper is two-fold. The first one is to prove that if an algebraic group is defined over a field $\mathbb{F}$ of characteristic zero, not necessarily algebraically closed, then any semisimple $\mathbb{F}$-automorphism keeps invariant a $\mathbb{F}$-Cartan subgroup and the maximal $\mathbb{F}$-torus contained in it (see Theorem 1.1). This extends a well-known result of R. Steinberg on the existence of an invariant maximal torus under a semisimple automorphism of an algebraic group over an algebraically closed field. The second goal is to obtain results on the surjectivity of the $n$-th power maps, $g \rightarrow g^{n}$, on algebraic groups which are not necessarily Zariski-connected (see Theorems 1.4 and 1.5) and on real algebraic groups which are not necessarily connected (see Theorems 1.7, 1.8, 1.9). One of our main motivations in investigating automorphism invariant Cartan subgroups is its application to the power maps of real algebraic groups (see Section 5).

Obtaining results on power maps is the primary goal of the earlier papers [Ch1], [Ch2] and [Ch3] and the part of the present article that deals with power maps is a natural continuation of these earlier works. We briefly recall the results obtained before in order to give a proper perspective to the present article. In [Ch2] the author has given a necessary and sufficient condition for the surjectivity of the $n$-th power maps for Zariski-connected algebraic groups over algebraically closed fields of characteristic zero; as an application a complete characterization was found of the set of integers $n$ for which the $n$-th power maps are surjective for semisimple algebraic groups over algebraically closed fields of characteristic zero. The author in [Ch3] and R. Steinberg in $[\mathrm{St}]$ independently extended the latter results of [Ch2] to semisimple algebraic groups over algebraically closed fields of arbitrary characteristic. The reader is further referred

To appear in Mathematische Zeitschrift

2000 Mathematics Subject Classification. Primary 20G15; Secondary 20G20.

Key words and phrases. Cartan subgroups of algebraic groups, power maps, surjectivity, weak-exponentiality. 
to [Ch1] for the results proved on the surjectivity of power maps on real solvable Lie groups and to [Ch4] and [Ch5] for more recent results on $p$-adic and real algebraic groups, respectively.

An automorphism $\psi$ of an algebraic group $G$ is called semisimple if the derivative $\mathrm{d} \psi$ is a semisimple linear map on its Lie algebra (see [St1] Section 7, pages 27-28). A crucial fact needed in Steinberg's paper [St], is his well-known result on the existence of a (semisimple) automorphism-invariant maximal torus in an algebraic group over an algebraically closed field (Theorem 7.5, [St1]). In order to prove results on the power maps of real algebraic groups, as done in Section 5, we require an analogue of the above result for such groups and in the following theorem we show that this holds in a much more general context, namely, for arbitrary algebraic groups defined over fields of characteristic zero.

For an algebraic group $G$, let $G^{0}$ denote the Zariski-connected component of the identity element. A group $\Gamma$ is called super-solvable if there are normal subgroups, $(e)=\Gamma_{0} \subset \cdots \subset$ $\Gamma_{k}=\Gamma$ such that the quotient $\Gamma_{i} / \Gamma_{i-1}$ is cyclic, for all $i$. We now state the first main result of this paper, the proof of which appears in Section 3.

Theorem 1.1. Let $G$ be an algebraic group defined over a field $\mathbb{F}$ of characteristic zero, not necessarily algebraically closed, and let $\Gamma$ be a super-solvable group of semisimple $\mathbb{F}$-automorphisms of $G$. Then there exists a Cartan subgroup $H$ of $G^{0}$, defined over $\mathbb{F}$, which remains invariant under $\Gamma$ i.e. $\psi(H)=H$, for all $\psi \in \Gamma$. In particular, there is a maximal torus $T$, defined over $\mathbb{F}$, with $\psi(T)=T$, for all $\psi \in \Gamma$.

Remark 1.2. In Theorem 1.1, $G$ is an arbitrary algebraic group and $\mathbb{F}$ is any field of characteristic zero. If $G$ is further assumed to be reductive then the above result remains valid for $\mathbb{F}$ of arbitrary characteristic, with the additional assumption that $\Gamma$ is a finite group of order prime to the characteristic of $\mathbb{F}$, as proved by Gopal Prasad in $[\mathrm{P}]$ and J.-P. Serre in $[\mathrm{S}]$ (see Theorem $3^{\prime \prime}$, Section 3.3. in $[\mathrm{S}]$ ). Thus the result of Prasad and Serre, and Theorem 1.1 complement each other.

Remark 1.3. It is proved in [B-S] that if $G$ is a compact real Lie group and $\Gamma$ is a super-solvable subgroup of $G$ then there is a $\Gamma$-stable maximal (compact) torus in $G$. One may view Theorem 1.1 as an analogue of the above result of $[\mathrm{B}-\mathrm{S}]$ in the algebraic group set-up. On the other hand, Theorem 1.1 can also be thought of as a generalisation of the above result in [B-S] because any compact real Lie group $G$ is of the form $H(\mathbb{R})$ for some algebraic group $H$, defined over $\mathbb{R}$ and if $T$ is a maximal torus over $\mathbb{R}$ in $H$ then $T(\mathbb{R})$ is a maximal (compact) torus of $G$.

Let $G$ be a group and let $P_{n}$ be the $n$-th power map defined by $P_{n}(g)=g^{n}$, for all $g \in G$. The following theorem, which we prove using certain results of [St], extends Theorem A of [Ch2] on power maps of Zariski-connected algebraic groups.

Theorem 1.4. Let $G$ be an algebraic group defined over an algebraically closed field of characteristic zero, which is not necessarily Zariski-connected, and let $n$ be an integer. Then $P_{n}: G \rightarrow G$ is surjective if and only if $n$ is prime to the order of the finite group $G / G^{0}$ and $P_{n}: G^{0} \rightarrow G^{0}$ is surjective. 
See Section 4 for a proof of the above theorem. In the light of Theorem A of [Ch2] we may rephrase Theorem 1.4 as follows.

Theorem 1.5. Let $G$ be an algebraic group defined over an algebraically closed field of characteristic zero, which is not necessarily Zariski-connected, and let $n$ be an integer. Let $T$ be a maximal torus in $G^{0}$. Then $P_{n}: G \rightarrow G$ is surjective if and only if $n$ is relatively prime to the orders of the finite groups $G / G^{0}$ and $Z_{T}(X) / Z_{T}(X)^{0}$, for all nilpotent elements $X$ in the Lie algebra $L(G)$ of $G$.

In the above theorem $Z_{T}(X)$ denotes the subgroup of elements of $T$ which fix $X \in L(G)$, through the adjoint action of $T$ on $L(G)$. We note that the orders of groups $Z_{T}(X) / Z_{T}(X)^{0}$, as above, can be computed using the finite number of characters on $T$ that appear as roots in $G$ (see [Ch2]). This completes our understanding of the surjectivity problem for power maps on algebraic groups over an algebraically closed field of characteristic zero.

By a real algebraic group we mean the group of real points, $G(\mathbb{R})$ where $G$ is an algebraic group defined over $\mathbb{R}$. The connected component of $G(\mathbb{R})$ in the real topology is denoted by $G(\mathbb{R})^{*}$. A real Lie group $H$ is said to be weakly exponential if the image $\exp (L(H))$, of the Lie algebra $L(H)$ under the exponential map, exp : $L(H) \rightarrow H$ is dense in $H$ (in the real topology of $H$ ). Our next results are related to the power maps of real algebraic groups, the main theme of which circles around Theorem 1.7. In the course of the proof of this result we also obtain Theorem 1.6 on weak exponentiality of real algebraic groups, which is of independent interest.

Recall A. Borel's classification of weakly exponential real Lie groups which says that a connected real Lie group $G$ is weakly exponential if and only if all the Cartan subgroups of $G$ are connected (see $[\mathrm{Ho}-\mathrm{M}]$ and $[\mathrm{Dj}-\mathrm{Ho}]$ ). The Theorem 1.6 may be regarded as an extension of Borel's result restricted to the class of groups $G(\mathbb{R})^{*}$ and on the other hand it clarifies the dependence of weak-exponentiality of such groups to the surjectivity of the square map $P_{2}$, on the set of semisimple elements. Note that any Cartan subgroup $H$ of $G(\mathbb{R})^{*}$ is of the form $H=G(\mathbb{R})^{*} \cap Z_{G^{0}}(T)$ for some maximal torus $T$ of $G^{0}$ which is defined over $\mathbb{R}$. For a subgroup $A$ of the algebraic group $G$ we denote the set of semisimple elements in $A$ by $\mathcal{S}(A)$.

Theorem 1.6. Let $G$ be an algebraic group defined over $\mathbb{R}$. Then the following are equivalent.

(1) $P_{2}: \mathcal{S}\left(G(\mathbb{R})^{*}\right) \rightarrow \mathcal{S}\left(G(\mathbb{R})^{*}\right)$ is surjective.

(2) All the Cartan subgroups of $G(\mathbb{R})^{*}$ are connected.

(3) If $T$ is any maximal torus in $G$, defined over $\mathbb{R}$, then $T(\mathbb{R})^{*}=G(\mathbb{R})^{*} \cap T(\mathbb{R})$.

(4) $G(\mathbb{R})^{*}$ is weakly exponential i.e., the set, $\exp (L(G(\mathbb{R})))$ is dense in $G(\mathbb{R})^{*}$ in the real topology.

Consequently, if $P_{2}: G(\mathbb{R})^{*} \rightarrow G(\mathbb{R})^{*}$ is surjective then $G(\mathbb{R})^{*}$ is weakly exponential.

See Section 5 for a proof of the above result. It should be noted that the converse of the last part of the above theorem does not hold in general (see Remark 5.4). 
In [Ch5] we have obtained various conditions for surjectivity of $P_{n}$ on Zariski connected maximal $(\mathbb{C})$-rank subgroups of real algebraic groups. Our next Theorem 1.7 yields a characterization of the surjectivity of the $n$-th power maps for real points of a real algebraic group which is not necessarily Zariski-connected. The proof of Theorem 1.7 is based on Theorem 5.5 which is established by applying Theorem 1.1 specifically to the case when $\mathbb{F}=\mathbb{R}$.

Theorem 1.7. Let $G$ be an algebraic group defined over $\mathbb{R}$, which is not necessarily Zariskiconnected, and let $n$ be an integer. Let $A$ be a subgroup of $G(\mathbb{R})$ with $G(\mathbb{R})^{*} \subset A \subset G(\mathbb{R})$. Then $P_{n}: A \rightarrow A$ is surjective if and only if for every unipotent element $u \in G(\mathbb{R})^{*}, n$ is relatively prime to the order of the finite group $Z_{A}(u) / Z_{A}(u)^{*}$ and $P_{n}: \mathcal{S}\left(Z_{A}(u)^{*}\right) \rightarrow \mathcal{S}\left(Z_{A}(u)^{*}\right)$ is surjective.

In other words,

(1) If $n$ is odd then $P_{n}: A \rightarrow A$ is surjective if and only if for every unipotent element $u \in G(\mathbb{R})^{*}, n$ is relatively prime to the order of the finite group $Z_{A}(u) / Z_{A}(u)^{*}$.

(2) $P_{2}: A \rightarrow A$ is surjective if and only if for every unipotent element $u \in G(\mathbb{R})^{*}$, the order of the finite group $Z_{A}(u) / Z_{A}(u)^{*}$ is odd and $Z_{A}(u)^{*}$ is weakly exponential.

As shown in Corollary 5.7, the above theorem generalises a result of Djoković and Thang, (see Theorem 2.2 of $[\mathrm{Dj}-\mathrm{T}]$ ) on the characterization of exponentiality of $G(\mathbb{R})^{*}$.

As a consequence of Theorem 1.7 we obtain Theorem 1.8 which can be viewed as an analogue of Theorem 1.4 in the realm of real algebraic groups.

Theorem 1.8. Let $G$ be an algebraic group over $\mathbb{R}$ and let $n$ be an integer. Then $P_{n}: G(\mathbb{R}) \rightarrow$ $G(\mathbb{R})$ is surjective if and only if $n$ is relatively prime to the order of $G(\mathbb{R}) / G(\mathbb{R})^{*}$ and $P_{n}$ : $G(\mathbb{R})^{*} \rightarrow G(\mathbb{R})^{*}$ is surjective.

A similar result is also proved in Corollary 5.8, regarding the power maps on $G(\mathbb{R})^{*}$, where $G$ is a Zariski-connected algebraic group over $\mathbb{R}$.

S.G. Dani and M. McCrudden in Theorem 2.4, [D-M] show that a real Lie group $G$, of the form $G=K \ltimes N$ (semidirect product) where $K$ is a (connected) compact subgroup and $N$ is a normal simply connected nilpotent subgroup, is exponential if and only if the centralizer $Z_{K}(v)$ is connected for all elements $v$ in $N$. As an application of Theorem 1.7 and Theorem 1.8 we prove Theorem 1.9 which generalises the above result of Dani and McCrudden.

Theorem 1.9. Let $K$ be a compact real Lie group (not necessarily connected) and $N$ be a simply connected nilpotent Lie group. Let $G=K \ltimes N$ and $n$ be an integer. Then $P_{n}: G \rightarrow G$ is surjective if and only if $n$ is coprime to the number of connected components of $K$ and $Z_{K^{*}}(v)$ for all $v \in N$.

The Theorems 1.7, 1.8, 1.9 and Corollary 5.8 are proved in Section 5. 


\section{NotATiOns}

The algebraic closure of a field $\mathbb{F}$ is denoted by $\overline{\mathbb{F}}$.

For an algebraic group (or a real Lie group) $E$ let $L(E)$ denote its Lie algebra. The Zariski connected component of identity in an algebraic group $G$ is denoted by $G^{0}$. If $H$ is a real Lie group the connected component of identity of $H$, in the real topology, is denoted by $H^{*}$.

For a group $G$ and a subset $A \subset G$ we denote the subgroup of elements commuting with all the elements of $A$, by $Z_{G}(A)$. In case $G$ is an algebraic group (or a Lie group) and $A \subset L(G)$ then we denote the subgroup of elements of $G$ which fix all the elements of $A$ via the adjoint representation, by $Z_{G}(A)$.

If $G$ is an algebraic group defined over a field $\mathbb{F}$ then the Lie algebra $L(G)$ acquires a $\mathbb{F}$ structure compatible with the $\mathbb{F}$-structure of $G$; the group of $\mathbb{F}$-rational points in $G$ and in $L(G)$ are denoted by $G(\mathbb{F})$ and $L(G)(\mathbb{F})$, respectively. For a subgroup $H$ of an algebraic group $G$ we denote the set of semisimple elements in $H$ by $\mathcal{S}(H)$.

\section{EXISTENCE OF AUTOMORPHISM INVARIANT CARTAN SUBGROUPS IN CHARACTERISTIC ZERO}

In this section we prove Theorem 1.1. We first need to recall certain basic definitions and facts. Recall that a Cartan subalgebra of a Lie algebra is a self normalizing nilpotent Lie subalgebra and according to C. Chevalley, a Cartan subgroup of an abstract group $G$ is a maximal nilpotent subgroup $H$ of $G$ so that any finite index normal subgroup of $H$ is of finite index in its own normalizer. It is well-known that Cartan subgroups of a Zariski-connected algebraic group are Zariski-closed, Zariski-connected, nilpotent groups and they are precisely the centralizers of some maximal torus in the ambient algebraic group. The next theorem is a collection of standard facts on algebraic groups.

Theorem 3.1. (Propositions 24.3.6, 24.3.5,29.2.5, [T-Y]) Let $G$ be a Zariski connected algebraic group (over an algebraically closed field of characteristic zero).

(1) Let $A$ be a Zariski connected algebraic subgroup of $G$. Then $Z_{G}(A)=Z_{G}(L(A))$. Further, if $v \in L(G)$ then $L\left(Z_{A}(v)\right)=Z_{L(A)}(v)$.

(2) Let $\mathfrak{h} \subset L(G)$ be a Cartan subalgebra. Then there exists a unique Cartan subgroup $H \subset G$ such that $L(H)=\mathfrak{h}$.

Lemma 3.2. Let $G$ be an algebraic group defined over a field $\mathbb{F}$, which is not necessarily algebraically closed. Let $\mathfrak{h}$ be a $\mathbb{F}$-Cartan subalgebra of $L(G)(\mathbb{F})$. Then there exists a unique Cartan subgroup $H \subset G$, defined over $\mathbb{F}$, so that $L(H)=\mathfrak{h} \otimes_{\mathbb{F}} \overline{\mathbb{F}}$.

Proof. Let $L(G)=\mathfrak{g}$ and $\overline{\mathfrak{h}}=\mathfrak{h} \otimes_{\mathbb{F}} \overline{\mathbb{F}}$. Then $\overline{\mathfrak{h}}$ is a $\overline{\mathbb{F}}$-Cartan subalgebra of $L(G)$. We recall that the subset of $\overline{\mathfrak{h}}$, consisting of points $z$ with the property that $\overline{\mathfrak{h}}=\left\{y \in \mathfrak{g} \mid(\operatorname{ad} z)^{\operatorname{dim} \mathfrak{g}} y=0\right\}$, is a nonempty Zariski open set in $\overline{\mathfrak{h}}$. 
By Theorem $3.1(2)$, there is a Zariski closed subgroup $H$ of $G$ such that $L(H)=\overline{\mathfrak{h}}$. Clearly $L(H)$ is defined over $\mathbb{F}$. Note that $L(H)(\mathbb{F})=\mathfrak{h}$ and hence $\mathfrak{h}$ is Zariski dense in $L(H)=\overline{\mathfrak{h}}$. Thus there exists $\alpha \in \mathfrak{h}$ such that $\overline{\mathfrak{h}}=\left\{y \in \mathfrak{g} \mid(\operatorname{ad} \alpha)^{\operatorname{dim} \mathfrak{g}} y=0\right\}$. Let $\alpha=\alpha_{s}+\alpha_{n}$ be the additive Jordan decomposition of $\alpha$. Note that $\alpha_{s}, \alpha_{n} \in \mathfrak{h}$. It is easy to verify that

$$
\begin{aligned}
\overline{\mathfrak{h}} & =\left\{y \in \mathfrak{g} \mid(\operatorname{ad} \alpha)^{\operatorname{dim} \mathfrak{g}} y=0\right\} \\
& =\left\{y \in \mathfrak{g} \mid\left(\operatorname{ad} \alpha_{s}\right) y=0\right\} .
\end{aligned}
$$

Let $H^{\prime}$ be the Zariski connected component of the algebraic subgroup $\left\{g \in G \mid \operatorname{Ad} g\left(\alpha_{s}\right)=\right.$ $\left.\alpha_{s}\right\}$. As $\alpha_{s} \in \mathfrak{h}=L(H)(\mathbb{F})$ we observe that $H^{\prime}$ is defined over $\mathbb{F}$. But by Theorem 3.1 (1), it follows that $H=H^{\prime}$. Thus $H$ is defined over $\mathbb{F}$. This completes the proof.

We next state a theorem due to Borel and Mostow (see Theorem 7.6 of $[\mathrm{B}-\mathrm{M}]$ ), which is one of the main ingredients in our proof of Theorem 1.1.

Theorem 3.3. (A. Borel and G.D. Mostow) Let $\mathfrak{g}$ be a Lie algebra over a characteristic zero field and $\Gamma$ be a super-solvable group of semisimple automorphisms of $\mathfrak{g}$. Then $\Gamma$ leaves a Cartan subalgebra of $\mathfrak{g}$ invariant.

We now prove Theorem 1.1.

Proof of Theorem 1.1. By the hypothesis $\psi: G \rightarrow G$ is a semisimple $\mathbb{F}$-automorphism, for all $\psi \in \Gamma$. Hence the $\mathbb{F}$-Lie algebra $L(G)(\mathbb{F})$ remains invariant under the derivative $\mathrm{d} \psi$, for all $\psi \in \Gamma$. Let $\widetilde{\Gamma}$ be the group generated by the semisimple automorphism $\mathrm{d} \psi$, for all $\psi \in \Gamma$. As $\widetilde{\Gamma}$ is super-solvable, by Theorem 3.3, there is a $\mathbb{F}$-Cartan subalgebra $\mathfrak{h}$ of $L(G)(\mathbb{F})$ which remains invariant under $\mathrm{d} \psi$, for all $\psi \in \Gamma$. By Lemma 3.2 there is a Cartan subgroup $H$ of $G^{0}$ which is defined over $\mathbb{F}$ so that $L(H)=\mathfrak{h} \otimes_{\mathbb{F}} \overline{\mathbb{F}}$. Clearly, $L(H)$ remains invariant under $\mathrm{d} \psi$, for all $\psi \in \Gamma$. Hence, by the uniqueness in Theorem $3.1(2), \psi(H)=H$, for all $\psi \in \Gamma$.

To prove the last part of Theorem 1.1 we recall that as $H$ is Zariski connected and nilpotent, there is a unique maximal torus $T \subset H$ which is central in $H$. As $H$ is a Cartan subgroup of $G$ it follows that $T$ is a maximal torus in $G$. Moreover as $H$ is defined over $\mathbb{F}$, so is $T$. Clearly $\psi(T)=T$, for all $\psi \in \Gamma$.

Corollary 3.4. Let $G$ be an algebraic group defined over a field $\mathbb{F}$ of characteristic zero, which is not necessarily algebraically closed. Let $s \in G(\mathbb{F})$ be a semisimple element. Then there exists a maximal $\mathbb{F}$-torus $T$ of $G^{0}$ so that $s T s^{-1}=T$.

Proof. The proof is immediate from Theorem 1.1 if we consider conjugation by $s$ as a $\mathbb{F}$ automorphism of $G^{0}$.

We conclude this section with some remarks and questions which are relevant to Theorem 1.1 . 
Remark 3.5. Let $\mathbb{F}$ be an arbitrary field, not necessarily algebraically closed and let $G$ be an algebraic group over $\mathbb{F}$ which is $\mathbb{F}$-isotropic i.e., $G$ admits a $\mathbb{F}$-split torus. Let $\psi$ be a semisimple $\mathbb{F}$-automorphism of $G$. Then it is not always true that $\psi$ will keep a $\mathbb{F}$-split torus of $G$ invariant. Following Gopal Prasad's suggestion, we can construct examples by considering an algebraic group $G$ which is $\mathbb{F}$-isotropic and which contains a maximal torus $T$ defined over $\mathbb{F}$ which is $\mathbb{F}$-anisotropic. A naive example of such a situation may be obtained by considering $\mathbb{F}=\mathbb{R}$ and $T=S O_{2}(\mathbb{C})$ and $G=S L_{2}(\mathbb{C})$. Note that $S O_{2}(\mathbb{C})$ is a $\mathbb{R}$-anisotropic maximal torus of the $\mathbb{R}$-algebraic group $S L_{2}(\mathbb{C})$. More generally, if $\mathbb{F}$ is an arbitrary field with a finite separable extension $\mathbb{K}$ of degree $n$, then the group, $\left\{x \in \mathbb{K}^{*} \mid \mathrm{N}_{\mathbb{K} / \mathbb{F}}(x)=1\right\}$ of norm one elements in $\mathbb{K}^{*}$ embeds in the $\mathbb{F}$-algebraic group $G=S L_{n}(\overline{\mathbb{F}})$ as the group of $\mathbb{F}$-rational points of a certain maximal $\mathbb{F}$-torus $T$ (called norm torus) which is $\mathbb{F}$-anisotropic in $G$. Being equipped with a $\mathbb{F}$-isotropic group $G$ with a maximal $\mathbb{F}$-torus $T \subset G$ which is $\mathbb{F}$-anisotropic, we first consider an element $\alpha \in T(\mathbb{F})$ such that the subgroup, $\langle\alpha\rangle$, generated by $\alpha$, is Zariski dense in $T$. We claim that $T$ is the unique maximal torus stable under $\psi_{\alpha}$, where $\psi_{\alpha}$ is the semisimple $\mathbb{F}$-automorphism of $G$ defined by $\psi_{\alpha}(g)=\alpha g \alpha^{-1}, g \in G$. To see this, let $T_{1}$ be another maximal torus such that $\psi_{\alpha}\left(T_{1}\right)=T_{1}$. As $\langle\alpha\rangle$ is Zariski dense in $T$ it follows that $T \subset N_{G}\left(T_{1}\right)$, where $N_{G}\left(T_{1}\right)$ is the normalizer of $T_{1}$ in $G$. As $T$ is connected and $G$ is reductive $T \subset Z_{G}\left(T_{1}\right)=T_{1}$. Since both $T_{1}, T$ are maximal we have $T=T_{1}$.

Remark 3.6. The above remark leads us to ask the following natural questions in the context of Prasad's and Serre's result ([P] and Theorem $3^{\prime \prime}$, Section 3.3. of [S], respectively), Steinberg's result (Theorem 7.5, [St1]), and Theorem 1.1. Let $G$ be an algebraic group over $\mathbb{F}$ and $\psi: G \rightarrow G$ be a semisimple $\mathbb{F}$-automorphism. It is of interest to know under what conditions $\psi$ will keep invariant a maximal $\mathbb{F}$-split torus of $G$. More generally, under what conditions on $\psi$, a minimal parabolic over $\mathbb{F}$ and a maximal $\mathbb{F}$-split torus contained in it, will remain $\psi$-invariant.

\section{Power maps on Disconnected algebraic groups over algebraically Closed FIELDS OF CHARACTERISTIC ZERO}

This section is devoted to proving Theorem 1.4. In the proof we do not use results from the previous section. However, we need the following theorem of R. Steinberg in [St], which is proved for algebraic groups over algebraically closed fields of arbitrary characteristic, but for our purpose we state it only for groups over algebraically closed fields of characteristic zero.

Theorem 4.1. (R. Steinberg) Let $H$ be an algebraic group over an algebraically closed field of characteristic zero and let $n$ be an integer. Then $P_{n}: \mathcal{S}(H) \rightarrow \mathcal{S}(H)$ is surjective if and only if $n$ is prime to the order of $H / H^{0}$.

We also need the following result; see Lemma 2.3 of [Ch2] for a proof.

Lemma 4.2. Let $G$ be an algebraic group. An element $g \in G$ is contained in $P_{n}(G)$ if and only if $g_{s} \in P_{n}\left(Z_{G}\left(g_{u}\right)\right)$. The map $P_{n}: G \rightarrow G$ is surjective if and only if for every unipotent element $u \in G$ the $\operatorname{map} P_{n}: \mathcal{S}\left(Z_{G}(u)\right) \rightarrow \mathcal{S}\left(Z_{G}(u)\right)$ is surjective. 
Proof of Theorem 1.4. Let $P_{n}: G \rightarrow G$ be surjective. It is immediate that $P_{n}: G / G^{0} \rightarrow G / G^{0}$ is surjective and hence $n$ is relatively prime to the order of $G / G^{0}$. Now let $g \in G^{0}$. Let $h \in G$ be such that $g=h^{n}$. This implies that in the group $G / G^{0}$ the element $\left(h G^{0}\right)^{n}=e$. As the order of $G / G^{0}$ is relatively prime to $n$, it follows that $h G^{0}=e$ and hence $h \in G^{0}$. Thus $P_{n}: G^{0} \rightarrow G^{0}$ is surjective.

We now prove the converse. Suppose that $n$ is relatively prime to the order of $G / G^{0}$ and that $P_{n}: G^{0} \rightarrow G^{0}$ is surjective. To prove that $P_{n}: G \rightarrow G$ is surjective, by Lemma 4.2 , it is enough to show that $P_{n}: \mathcal{S}\left(Z_{G}(u)\right) \rightarrow \mathcal{S}\left(Z_{G}(u)\right)$ is surjective, for every unipotent element $u \in G$. If $u \in G$ is unipotent then it lies in $G^{0}$. In view of Theorem 4.1 it is now enough to show that $n$ is prime to the order of the group $Z_{G}(u) / Z_{G}(u)^{0}$. Since $P_{n}: G^{0} \rightarrow G^{0}$ is surjective it follows by using Lemma 4.2 and Theorem 4.1 that $n$ is prime to the order of the finite group $Z_{G^{0}}(u) / Z_{G^{0}}(u)^{0}$. We have the inclusions

$$
Z_{G^{0}}(u)^{0} \subset Z_{G^{0}}(u) \subset Z_{G}(u) \text { and } Z_{G}(u)^{0} \subset Z_{G}(u) .
$$

Note that the connected groups $Z_{G^{0}}(u)^{0}$ and $Z_{G}(u)^{0}$ have the same Lie subalgebra in the Lie algebra of $G$ and hence $Z_{G^{0}}(u)^{0}=Z_{G}(u)^{0}$. So we have

$$
Z_{G^{0}}(u)^{0}=Z_{G}(u)^{0} \subset Z_{G^{0}}(u) \subset Z_{G}(u) .
$$

Note also that the group $Z_{G}(u) / Z_{G^{0}}(u)$ embeds in the group $G / G^{0}$. Hence $n$ is relatively prime to the order of $Z_{G}(u) / Z_{G^{0}}(u)$. Thus $n$ is relatively prime to the order of $Z_{G}(u) / Z_{G}(u)^{0}$. This completes the proof of the theorem.

\section{Power maps on Disconnected Real Algebraic Groups}

In this section we prove Theorems 1.6 and 1.8. To prove Theorem 1.8 we use Theorem 5.5, which can be thought of as an analogue of Theorem 4.1. We further remark that Corollary 3.4 in Section 3 is applied to deduce Theorem 5.5.

It can be easily verified that, for a group $G, P_{n}: G \rightarrow G$ is surjective if and only if $P_{m}: G \rightarrow G$ is surjective for all the factors $m$ of $n$. In view of this it is important to note that the surjectivity of power maps on groups can be divided into two cases, namely, surjectivity of $P_{n}$, when $n$ is odd and surjectivity of $P_{2}$.

We next state a result due to Borel and Tits (see [B-T], Theorem 14.4, page 146) which is particular to algebraic groups defined over $\mathbb{R}$.

Theorem 5.1. (A. Borel and J. Tits) Let $G$ be a Zariski connected algebraic group defined over $\mathbb{R}$. Then either $G(\mathbb{R})=G(\mathbb{R})^{*}$ or $G(\mathbb{R}) / G(\mathbb{R})^{*}$ is a direct product of cyclic groups of order two.

We note that if $T \subset G$ is a torus of $G$ defined over $\mathbb{R}$ then $T(\mathbb{R})^{*} \subset T(\mathbb{R}) \cap G(\mathbb{R})^{*}$. Hence by Theorem 5.1, either $T(\mathbb{R})^{*}=T(\mathbb{R}) \cap G(\mathbb{R})^{*}$ or $T(\mathbb{R}) \cap G(\mathbb{R})^{*} / T(\mathbb{R})^{*}$ is a direct product of cyclic groups of order two. We also need the following result. 
Lemma 5.2. Let $G$ be a Zariski connected algebraic group defined over $\mathbb{R}$. Let $T$ be a maximal torus of $G$ which is defined over $\mathbb{R}$. Suppose $T(\mathbb{R}) \cap G(\mathbb{R})^{*}$ is not connected (in the real topology). Then any non-trivial coset in the group $T(\mathbb{R}) \cap G(\mathbb{R})^{*} / T(\mathbb{R})^{*}$ contains an element $\beta$ such that $Z_{G}(\beta)=Z_{G}(T)$.

Proof. We first observe that if $H$ is a Zariski connected algebraic group over $\mathbb{R}$ and $S$ is a torus in $H$ which is defined over $\mathbb{R}$, then $S(\mathbb{R})^{*}$ is Zariski dense in $S$. This follows from the fact that $S(\mathbb{R})$ is Zariski dense in $S$ and $S(\mathbb{R})^{*}$ is a finite index subgroup of $S(\mathbb{R})$.

Now we get back to the proof of the lemma. Recall that, there exist finitely many non-trivial algebraic characters $\chi_{1}, \cdots, \chi_{n}$ of $T$ with the property that, if $t \in T$ satisfies $\chi(t) \neq 1$ for all $i=1, \cdots, n$, then $Z_{G}(t)=Z_{G}(T)$ (see [Sp], Lemma 6.4.3, page 109). Let $h T(\mathbb{R})^{*}$ be a nontrivial coset in $T(\mathbb{R}) \cap G(\mathbb{R})^{*}$. Choose any $\alpha \in h T(\mathbb{R})^{*}$. Consider the subset $A \subset T$ defined by,

$$
A=\left\{t \in T \mid \chi_{i}^{-1}(t) \neq \chi_{i}(\alpha) \text {, for all } i=1, \cdots, n\right\} .
$$

Clearly $A$ is non-empty and Zariski open in $T$. By the above observation $T(\mathbb{R})^{*}$ is Zariski dense in $T$ and hence we have a $t_{0} \in T(\mathbb{R})^{*}$ so that $\chi_{i}\left(\alpha t_{0}\right) \neq 1$ for all $i$. Thus $Z_{G}\left(\alpha t_{0}\right)=Z_{G}(T)$ with $\alpha t_{0} \in h T(\mathbb{R})^{*}$. Setting $\beta=\alpha t_{0}$ we see that $\beta$ has the desired properties .

Before giving a proof of Theorem 1.6, we state a result due to A. Borel on the classification of weakly exponential real Lie groups (see $[\mathrm{Ho}-\mathrm{M}]$ and $[\mathrm{Dj}-\mathrm{Ho}]$ ).

Theorem 5.3. (A. Borel) A connected real Lie group $G$ is weakly exponential if and only if all the Cartan subgroups of $G$ are connected.

Proof of Theorem 1.6. We begin by proving the implications $(2) \Leftrightarrow(3)$. We recall that, if $T$ is a maximal torus of $G$ over $\mathbb{R}$ then $Z_{G^{0}}(T)(\mathbb{R}) \cap G(\mathbb{R})^{*}$ is a Cartan subgroup of $G(\mathbb{R})^{*}$ and any Cartan subgroup of $G(\mathbb{R})^{*}$ is of this form for some maximal torus $T$ of $G$, which is defined over $\mathbb{R}$. We further note that, $Z_{G^{0}}(T)(\mathbb{R}) \cap G(\mathbb{R})^{*}=\left(T(\mathbb{R}) \cap G(\mathbb{R})^{*}\right) \times U(\mathbb{R})$ for some unipotent subgroup $U$ of $G$ and that $T(\mathbb{R})^{*}$ is of finite index in $T(\mathbb{R}) \cap G(\mathbb{R})^{*}$. Clearly $U(\mathbb{R})$ is connected. Hence $Z_{G^{0}}(T)(\mathbb{R}) \cap G(\mathbb{R})^{*}$ is connected if and only if $T(\mathbb{R}) \cap G(\mathbb{R})^{*}$ is connected. This in turn implies that, $Z_{G^{0}}(T)(\mathbb{R}) \cap G(\mathbb{R})^{*}$ is connected if and only if $T(\mathbb{R})^{*}=T(\mathbb{R}) \cap G(\mathbb{R})^{*}$, proving $(2) \Leftrightarrow(3)$.

Implications $(2) \Leftrightarrow(4)$ follows immediately from Theorem 5.3.

We now prove $(2) \Rightarrow(1)$. Let $s \in G(\mathbb{R})^{*}$ be a semisimple element. Hence $s$ lies in a Cartan subgroup $C$ of $G(\mathbb{R})^{*}$. Now $C$ is a connected nilpotent Lie group and hence $\exp (L(C))=C$. So $P_{2}: C \rightarrow C$ is surjective. Thus there exists $r \in C$ so that $s=r^{2}$.

We now show $(1) \Rightarrow(2)$. We assume that $P_{2}: \mathcal{S}\left(G(\mathbb{R})^{*}\right) \rightarrow \mathcal{S}\left(G(\mathbb{R})^{*}\right)$ is surjective. Let $C \subset G(\mathbb{R})^{*}$ be a Cartan subgroup. Then $C=Z_{G^{0}}(T)(\mathbb{R}) \cap G(\mathbb{R})^{*}$ for some maximal torus $T$, defined over $\mathbb{R}$. We will arrive at a contradiction by assuming that $C=Z_{G^{0}}(T)(\mathbb{R}) \cap G(\mathbb{R})^{*}$ is not connected in the real topology. By the implications $(2) \Leftrightarrow(3)$ we have that $T(\mathbb{R}) \cap G(\mathbb{R})^{*}$ is not 
connected in the real topology. Choose any non-trivial coset in the group $T(\mathbb{R}) \cap G(\mathbb{R})^{*} / T(\mathbb{R})^{*}$. By Lemma 5.2, this non-trivial coset contains an element $\beta$ such that $Z_{G}(\beta)=Z_{G}(T)$. We will show that $\beta$ has no 2 -th root in $G(\mathbb{R})^{*}$. If $\gamma \in G(\mathbb{R})^{*}$ is such that $\gamma^{2}=\beta$ then $\gamma \in$ $Z_{G}(\beta)=Z_{G}(T)$. As $\gamma$ is semisimple we have that $\gamma \in T(\mathbb{R}) \cap G(\mathbb{R})^{*}$. But as $\gamma^{2}=\beta$, it follows that $\beta \in T(\mathbb{R})^{*}$. This is contradiction to the fact that $\beta$ lies in a non-trivial coset of $T(\mathbb{R}) \cap G(\mathbb{R})^{*} / T(\mathbb{R})^{*}$. This completes the proof of the theorem.

Remark 5.4. The Theorem 1.6 says that surjectivity of $P_{2}: G(\mathbb{R})^{*} \rightarrow G(\mathbb{R})^{*}$ implies weakexponentiality of $G(\mathbb{R})^{*}$. However, the converse is not true. If $G$ is the Weil restriction $\mathcal{R}_{\mathbb{C} / \mathbb{R}}\left(S O_{n}(\mathbb{C})\right)$, then $G$ is a group over $\mathbb{R}$ with $G(\mathbb{R})^{*}=G(\mathbb{R})=S O_{n}(\mathbb{C})$. By Theorem $\mathrm{C}$ of $[\mathrm{Ch} 2]$, for every $n \geq 3$, the map $P_{2}: S O_{n}(\mathbb{C}) \rightarrow S O_{n}(\mathbb{C})$ is not surjective. Thus $P_{2}: G(\mathbb{R})^{*} \rightarrow G(\mathbb{R})^{*}$ is not surjective. But, as $G(\mathbb{R})^{*}$ is a connected complex Lie group, the Cartan subgroups of $G(\mathbb{R})^{*}$ are connected and hence by Theorem $5.3, G(\mathbb{R})^{*}$ is weakly exponential.

In the proof of the following theorem we will apply Corollary 3.4 to algebraic groups over $\mathbb{R}$. The technique of our proof of this theorem is inspired by R. Steinberg's proof of Theorem 4.1 in [St]. A subgroup $A$ of an algebraic group $G$ is said to be closed under Jordan-decomposition if for all $g \in A$, both the semisimple part $g_{s}$ and the unipotent part $g_{u}$ lie in $A$. Note that if $G$ is an algebraic group over $\mathbb{R}$ then $G(\mathbb{R}) \subset G$ is closed under Jordan decomposition (see [Sp]). Moreover, as any unipotent element $u \in G(\mathbb{R})$ lies in $G(\mathbb{R})^{*}$, it follows that if $A$ is a subgroup of $G$ with $G(\mathbb{R})^{*} \subset A \subset G(\mathbb{R})$ then $A$ is closed under Jordan decomposition.

Theorem 5.5. Let $H$ be an algebraic group over $\mathbb{R}, A$ be a subgroup of $H$ with $H(\mathbb{R})^{*} \subset A \subset$ $H(\mathbb{R})$ and let $n$ be an integer. Then $P_{n}: \mathcal{S}(A) \rightarrow \mathcal{S}(A)$ is surjective if and only if $n$ is relatively prime to the order of the finite group $A / H(\mathbb{R})^{*}$ and $P_{n}: \mathcal{S}\left(H(\mathbb{R})^{*}\right) \rightarrow \mathcal{S}\left(H(\mathbb{R})^{*}\right)$ is surjective.

In other words,

(1) If $n$ is an odd integer. Then $P_{n}: \mathcal{S}(A) \rightarrow \mathcal{S}(A)$ is surjective if and only if $n$ is relatively prime to the order of the finite group $A / H(\mathbb{R})^{*}$.

(2) $P_{2}: \mathcal{S}(A) \rightarrow \mathcal{S}(A)$ is surjective if and only if the order of the finite group $A / H(\mathbb{R})^{*}$ is odd and $P_{2}: \mathcal{S}\left(H(\mathbb{R})^{*}\right) \rightarrow \mathcal{S}\left(H(\mathbb{R})^{*}\right)$ surjective. Furthermore, this happens if and only if the order of the finite group $A / H(\mathbb{R})^{*}$ is odd and $H(\mathbb{R})^{*}$ is weakly exponential.

Proof. The proof will be given in three steps. The Step 1 is of general interest. In Steps 2 and 3 we prove the first part of the theorem. The last two (numbered) parts, the proofs of which we skip, are essentially a restatement of the first part of the theorem and they can be readily proved using the first part and Step 1.

Step 1: Observe that if $n$ is an odd integer and $H$ is an algebraic group over $\mathbb{R}$ then $P_{n}$ : $\mathcal{S}\left(H(\mathbb{R})^{*}\right) \rightarrow \mathcal{S}\left(H(\mathbb{R})^{*}\right)$ is surjective. To see this first note that $H(\mathbb{R})^{*} \subset H^{0}$. Let $x$ be a semisimple element in $H(\mathbb{R})^{*}$. Then there is a maximal $\mathbb{R}$-torus $T \subset H^{0}$ such that $x \in T(\mathbb{R})$. Clearly, $T(\mathbb{R})^{*}$ is a finite index subgroup of $T(\mathbb{R}) \cap H(\mathbb{R})^{*}$ with the index being a power of 2 . 
As $n$ is odd, $T(\mathbb{R})$ is abelian and $T(\mathbb{R})^{*}$ is connected, it follows that there is a $y \in T(\mathbb{R}) \cap H(\mathbb{R})^{*}$ such that $x=y^{n}$. Thus $P_{n}: \mathcal{S}\left(H(\mathbb{R})^{*}\right) \rightarrow \mathcal{S}\left(H(\mathbb{R})^{*}\right)$ is surjective if $n$ is odd.

Step 2: Note that it is enough to prove the theorem when $n$ is a prime number. We first prove the "if" part. Suppose $n$ is relatively prime to the order of the finite group $A / H(\mathbb{R})^{*}$ and $P_{n}: \mathcal{S}\left(H(\mathbb{R})^{*}\right) \rightarrow \mathcal{S}\left(H(\mathbb{R})^{*}\right)$ is surjective. In view of Step 1 this is equivalent to assuming that $n$ is relatively prime to the order of $A / H(\mathbb{R})^{*}$ when $n$ is an odd prime and further, when $n=2$ the order of $A / H(\mathbb{R})^{*}$ is odd and $P_{2}: \mathcal{S}\left(H(\mathbb{R})^{*}\right) \rightarrow \mathcal{S}\left(H(\mathbb{R})^{*}\right)$ is surjective.

Let $s \in A$ be a semisimple element and suppose that the order of $s H(\mathbb{R})^{*}$ in the finite group $A / H(\mathbb{R})^{*}$ is $m$. Clearly $m$ is relatively prime to $n$. Now as $s^{m} \in H(\mathbb{R})^{*}$ it follows that $s^{m} \in H^{0}$. Hence $s^{m} \in Z_{H}\left(s^{m}\right)^{0}$. Let $H^{\prime}=Z_{H}\left(s^{m}\right)$. Then $H^{\prime}$ is an algebraic group defined over $\mathbb{R}$ and $s \in H^{\prime}(\mathbb{R})$ and $s^{m} \in\left(H^{\prime}\right)^{0}(\mathbb{R})$. We now apply Corollary 3.4 to the situation when the underlying field is $\mathbb{R}$ and get that there is a maximal $\mathbb{R}$-torus $T \subset H^{\prime}$ so that $s T s^{-1}=T$.

Claim: $P_{n}: Z_{T(\mathbb{R})^{*}}(s) \rightarrow Z_{T(\mathbb{R})^{*}}(s)$ is surjective.

Note that $s T(\mathbb{R}) s^{-1}=T(\mathbb{R})$ and hence $s T(\mathbb{R})^{*} s^{-1}=T(\mathbb{R})^{*}$. As $s^{m} \in\left(H^{\prime}\right)^{0}$ and $s^{m}$ is central in $H^{\prime}$ we get that $s^{m} \in T$. Let $\alpha: T(\mathbb{R})^{*} \rightarrow T(\mathbb{R})^{*}$ denote the conjugation in $T(\mathbb{R})^{*}$ defined by $\alpha(y)=$ sys $^{-1}, y \in T(\mathbb{R})^{*}$. Let $x \in Z_{T(\mathbb{R})^{*}}$. As $T(\mathbb{R})^{*}$ is a connected abelian group there is $t_{0} \in T(\mathbb{R})^{*}$ so that $t_{0}^{n}=x$. Now consider the element $t_{1}=t_{0} \alpha\left(t_{0}\right) \cdots \alpha^{m-1}\left(t_{0}\right)$. Clearly $\alpha\left(t_{1}\right)=t_{1}$ and hence $t_{1} \in Z_{T(\mathbb{R})^{*}}(s)$. Moreover $t_{1}{ }^{n}=x^{m}$. As $m$ and $n$ are relatively prime there are integers $a$ and $b$ so that $a m+b n=1$. As $t_{1}$ and $x$ commute, we have $x=x^{a m} x^{b n}=$ $t_{1}{ }^{a n} x^{b n}=\left(t_{1}{ }^{a} x^{b}\right)^{n}$. Clearly $t_{1}^{a} x^{b} \in Z_{T(\mathbb{R})^{*}}(s)$. This completes the proof of the claim.

We now consider two cases.

Case 1: Assume that $n$ is an odd prime. Note that $s^{m} \in T(\mathbb{R})$ and the group $T(\mathbb{R}) / T(\mathbb{R})^{*}$ is a product of cyclic groups of order two. Thus, $s^{2 m} \in T(\mathbb{R})^{*}$ and consequently $s^{2 m} \in Z_{T(\mathbb{R})^{*}}(s)$. By the above claim there is a $t \in Z_{T(\mathbb{R})^{*}}(s)$ so that $t^{n}=s^{2 m}$. Moreover as $n$ is odd and $m, n$ are relatively prime, the integers $2 m, n$ are also relatively prime. Hence there are integers $c, d$ with $c(2 m)+d n=1$. Now $s=s^{2 m c} s^{d n}=t^{c n} s^{d n}=\left(t^{c} s^{d}\right)^{n}$.

Case 2: Assume that $n=2$. By the assumption we have that $P_{2}: \mathcal{S}\left(H(\mathbb{R})^{*}\right) \rightarrow \mathcal{S}\left(H(\mathbb{R})^{*}\right)$ is surjective and the order of the finite group $H(\mathbb{R}) / H(\mathbb{R})^{*}$ is an odd integer. Hence by Theorem 1.6, $T(\mathbb{R})^{*}=T(\mathbb{R}) \cap H(\mathbb{R})^{*}$. We note that $s^{m} \in T(\mathbb{R}) \cap H(\mathbb{R})^{*}=T(\mathbb{R})^{*}$ and hence $s^{m} \in$ $Z_{T(\mathbb{R})^{*}}(s)$. Now the proof follows on the same lines as in the Case 1. By the above claim there is a $t \in Z_{T(\mathbb{R})^{*}}(s)$ so that $t^{2}=s^{m}$. Moreover, as the order of the finite group $A / H(\mathbb{R})^{*}$ is odd, the integer $m$ is also odd. Hence there is an integers $d$ with $m+2 d=1$. Now $s=s^{m} s^{2 d}=$ $t^{2} s^{2 d}=\left(t s^{d}\right)^{2}$.

Step 3: To prove the "only if" part we observe that every coset of $A / H(\mathbb{R})^{*}$ is represented by semisimple elements in $\mathcal{S}(A)$. Thus the surjectivity of $P_{n}: \mathcal{S}(A) \rightarrow \mathcal{S}(A)$ implies the surjectivity of $P_{n}: A / H(\mathbb{R})^{*} \rightarrow A / H(\mathbb{R})^{*}$ and hence the order of $A / H(\mathbb{R})^{*}$ is relatively prime to $n$. It also follows easily that $P_{n}: \mathcal{S}\left(H(\mathbb{R})^{*}\right) \rightarrow \mathcal{S}\left(H(\mathbb{R})^{*}\right)$ is surjective. 
Lemma 5.6. Let $G$ be an algebraic group defined over $\mathbb{R}$. Let $A$ be a subgroup of $G(\mathbb{R})$ with $G(\mathbb{R})^{*} \subset A \subset G(\mathbb{R})$. Then $P_{n}: A \rightarrow A$ is surjective if and only if for every unipotent element $u \in G(\mathbb{R})^{*}$ the map $P_{n}: \mathcal{S}\left(Z_{A}(u)\right) \rightarrow \mathcal{S}\left(Z_{A}(u)\right)$ is surjective.

Proof. Note that any unipotent element $u$ in $G(\mathbb{R})$ belongs to a one parameter subgroup of $G(\mathbb{R})$, which is in turn contained in $Z_{G(\mathbb{R})^{*}}(u)$. Thus any unipotent element $u$ admits a $n$-th root in $Z_{G(\mathbb{R})^{*}}(u)$. Now recall that $A \subset G(\mathbb{R})$ is closed under Jordan decomposition. The proof is now similar to that of Lemma 2.3 in [Ch2] and hence we omit the details.

Proof of Theorem 1.7. The proof follows immediately from Theorem 5.5 and Lemma 5.6.

The result of Djoković and Thang (see Theorem 2.2 of $[\mathrm{Dj}-\mathrm{T}]$ ) on the characterization of exponentiality of $G(\mathbb{R})^{*}$ can be reproved using Theorem 1.7.

Corollary 5.7. (D. Z. Djoković and N. Q. Thang) Let $G$ be an algebraic group over $\mathbb{R}$. Then $\exp : \mathrm{L}(\mathrm{G}(\mathbb{R})) \rightarrow \mathrm{G}(\mathbb{R})^{*}$ is surjective if and only if $Z_{G(\mathbb{R})^{*}}(u)$ is weakly exponential for every unipotent element $u \in G(\mathbb{R})^{*}$.

Proof. It follows from $[\mathrm{M}]$ that $\exp : \mathrm{L}(\mathrm{G}(\mathbb{R})) \rightarrow \mathrm{G}(\mathbb{R})^{*}$ is surjective if and only if $P_{n}$ : $G(\mathbb{R})^{*} \rightarrow G(\mathbb{R})^{*}$ is surjective for all $n$. Using Theorem 1.7 , we conclude that this happens if and only if $Z_{G(\mathbb{R})^{*}}(u)=Z_{G}(u)(\mathbb{R})^{*}$ and $Z_{G}(u)(\mathbb{R})^{*}$ is weakly exponential for all unipotent elements $u \in G(\mathbb{R})^{*}$. This completes the proof.

We now prove Theorem 1.8

Proof of Theorem 1.8. We first prove the "if" part. Suppose $n$ is relatively prime to the order of $G(\mathbb{R}) / G(\mathbb{R})^{*}$ and $P_{n}: G(\mathbb{R})^{*} \rightarrow G(\mathbb{R})^{*}$ is surjective. Let $u \in G(\mathbb{R})$ be unipotent. Clearly $u \in G(\mathbb{R})^{*}$. Let $H_{u}=Z_{G(\mathbb{R})^{*}}(u)$. By assumption, $P_{n}: G(\mathbb{R})^{*} \rightarrow G(\mathbb{R})^{*}$ is surjective. Hence by Lemma 5.6, the map $P_{n}$ is surjective on the semisimple elements of $Z_{G(\mathbb{R})^{*}}(u)=H_{u}$. Observe that as all the unipotent elements of $H_{u}$ lie in $H_{u}^{*}$, every coset of the finite group $H_{u} / H_{u}^{*}$ is represented by semisimple elements in $H_{u}$. So it follows that the map $P_{n}$ is surjective on the group $H_{u} / H_{u}^{*}$. Hence $n$ is relatively prime to the order of $H_{u} / H_{u}^{*}$. Moreover, it also follows that $P_{n}: \mathcal{S}\left(H_{u}^{*}\right) \rightarrow \mathcal{S}\left(H_{u}^{*}\right)$ is surjective. As $H_{u}^{*}=Z_{G}(u)(\mathbb{R})^{*}$, we have that $P_{n}: \mathcal{S}\left(Z_{G}(u)(\mathbb{R})^{*}\right) \rightarrow$ $\mathcal{S}\left(Z_{G}(u)(\mathbb{R})^{*}\right)$ is surjective. Recall that $H_{u}^{*}=Z_{G}(u)(\mathbb{R})^{*} \subset Z_{G(\mathbb{R})^{*}}(u)=H_{u} \subset Z_{G(\mathbb{R})}(u)$. Note that the group $Z_{G(\mathbb{R})}(u) / Z_{G(\mathbb{R})^{*}}(u)$ embeds in the group $G(\mathbb{R}) / G(\mathbb{R})^{*}$ and hence $n$ is relatively prime to the order of the group $Z_{G(\mathbb{R})}(u) / Z_{G(\mathbb{R})^{*}}(u)$. We have shown above that $n$ is relatively prime to the order of $H_{u} / H_{u}^{*}=Z_{G(\mathbb{R})^{*}}(u) / Z_{G}(u)(\mathbb{R})^{*}$. Hence $n$ is relatively prime to the order of the group $Z_{G(\mathbb{R})^{*}}(u) / Z_{G}(u)(\mathbb{R})^{*}$. Now by Theorem 1.7 we see that $P_{n}$ is surjective on $G(\mathbb{R})$.

The proof of the "only if" part is straightforward and we omit it.

Corollary 5.8. Let $G$ be a Zariski connected algebraic group over $\mathbb{R}$. Let $n$ be an odd integer. Then $P_{n}: G(\mathbb{R}) \rightarrow G(\mathbb{R})$ is surjective if and only if $P_{n}: G(\mathbb{R})^{*} \rightarrow G(\mathbb{R})^{*}$ is surjective. 
Proof. The proof follows immediately from Theorem 1.8 and the fact that for a Zariskiconnected complex algebraic group $G$ over $\mathbb{R}$, the group $G(\mathbb{R}) / G(\mathbb{R})^{*}$ is a product of cyclic groups of order two (see Theorem 5.1).

Remark 5.9. We keep the notations as in Theorem 1.7. In view of Theorem 1.8 the question of surjectivity of $P_{n}$ on $G(\mathbb{R})$ reduces to that of $G(\mathbb{R})^{*}$. For each unipotent element $u \in G(\mathbb{R})^{*}$ we choose a maximal compact subgroup $C_{u}$ of $Z_{G(\mathbb{R})^{*}}(u)$. Then it follows from Theorem 3.1, page 180-181, [H] that the groups $Z_{G(u)^{*}}(\mathbb{R}) / Z_{G}(u)(\mathbb{R})^{*}$ and $C_{u} / C_{u}^{*}$ are isomorphic. Thus, by Theorem 1.7, if $n$ is odd then $P_{n}: G(\mathbb{R})^{*} \rightarrow G(\mathbb{R})^{*}$ is surjective if and only if $n$ is coprime to the orders of groups $C_{u} / C_{u}^{*}$ for all unipotent elements $u \in G(\mathbb{R})^{*}$. Further, $P_{2}: G(\mathbb{R})^{*} \rightarrow G(\mathbb{R})^{*}$ is surjective if and only if order of $C_{u} / C_{u}^{*}$ is odd and $Z_{G(u)^{*}}(\mathbb{R})^{*}$ is weakly exponential for unipotent elements $u \in G(\mathbb{R})^{*}$.

Further, if $G$ is a semisimple group over $\mathbb{R}$ one can say more using the theory of real nilpotent orbits as in Chapter 9, [Co-M]. Let $\theta$ be a Cartan involution of $G(\mathbb{R})^{*}$ and $K$ be the corresponding maximal compact subgroup, i.e. $K=\left\{g \in G(\mathbb{R})^{*} \mid \theta(g)=g\right\}$. A triple $\{H, X, Y\} \subset L(G(\mathbb{R}))$ is called a Cayley triple if it is a sl 2 -triple i.e. $[H, X]=2 X,[H, Y]=-2 Y,[X, Y]=H$ and moreover, if $\theta(H)=-H, \theta(X)=-Y$. Then it follows from Remark 9.5.2, page 148, [Co-M] that the collection of finite groups $C_{u} / C_{u}^{*}$ with $u$ varying over the unipotent elements in $G(\mathbb{R})^{*}$ is the same as the collection of finite groups $Z_{K}(H, X, Y) / Z_{K}(H, X, Y)^{*}$ with $\{H, X, Y\}$ varying over the set of Cayley triples of $L(G(\mathbb{R}))$. Thus if $n$ is odd then $P_{n}: G(\mathbb{R})^{*} \rightarrow G(\mathbb{R})^{*}$ is surjective if and only if $n$ is coprime to the orders of the finite groups $Z_{K}(H, X, Y) / Z_{K}(H, X, Y)^{*}$ for all Cayley triples $\{H, X, Y\}$ of $L(G(\mathbb{R}))$.

We now proceed towards the proof of Theorem 1.9. We need the following lemma.

Lemma 5.10. Let $G$ be a real Lie group with a compact subgroup $K$ and a simply connected nilpotent normal subgroup $N$ such that $G=K \ltimes N$. Then, for every $v \in N$ the subgroup $Z_{K}(v)$ is a maximal compact subgroup of $Z_{G}(v)$.

Proof. We first consider the case when $N$ is abelian. Let $K_{1}$ be a maximal compact subgroup of $Z_{G}(v)$ where $v \in N$. Then, as $G$ has finitely many connected components, by Theorem 3.1, page 180-181, [H] there is an $\alpha \in G$ such that $\alpha K_{1} \alpha^{-1} \subset K$. We may choose $\alpha \in N$. Now, as $N$ is abelian, we have,

$$
\alpha K_{1} \alpha^{-1} \subset \alpha Z_{G}(v) \alpha^{-1}=Z_{G}(v) .
$$

Thus $\alpha K_{1} \alpha^{-1}$ is another maximal compact subgroup of $Z_{G}(v)$ and further $\alpha K_{1} \alpha^{-1} \subset Z_{K}(v) \subset$ $Z_{G}(v)$. Hence $Z_{K}(v)$ is a maximal compact subgroup of $Z_{G}(v)$.

We next consider the case when $N$ is not necessarily abelian. Let $N^{\prime}=N /[N, N]$. Now consider the group $G^{\prime}=K \ltimes N^{\prime}$. Let $\pi: G \rightarrow G^{\prime}$ be the natural quotient homomorphism. Let $v \in N$, as before, and let $K_{2} \subset Z_{G}(v)$ be a maximal compact subgroup of $Z_{G}(v)$ such that $Z_{K}(v) \subset K_{2}$. We will show that $Z_{K}(v)=K_{2}$. Let $v^{\prime}=\pi(v)$. As $N^{\prime}$ is abelian, using the first case, we see that $Z_{K}\left(v^{\prime}\right)$ is a maximal compact subgroup of $Z_{G^{\prime}}\left(v^{\prime}\right)$ and consequently 
$\pi\left(K_{2}\right)=\pi\left(Z_{K}(v)\right)$. Now as $[N, N]$ is simply connected nilpotent, $[N, N] \cap K_{2}$ is trivial. This, together with the equality $\pi\left(K_{2}\right)=\pi\left(Z_{K}(v)\right)$, implies that $K_{2}=Z_{K}(v)$.

Proof of Theorem 1.9. We first note a standard fact that if $G=K \ltimes N$ then there is an algebraic group $H$ over $\mathbb{R}$ and a Lie group isomorphism $\phi: G \rightarrow H(\mathbb{R})$. It also follows that there is a Levi subgroup $L$ of $H$ such that $\phi(K)=L(\mathbb{R})$ and $\phi(N)=R_{u} H(\mathbb{R})$. Clearly, as $L(\mathbb{R})$ is compact, any unipotent element $u \in H(\mathbb{R})$ is contained in $R_{u} H(\mathbb{R})$.

We next observe that if $H$ is as above and if $H_{1}$ is any algebraic subgroup of $H$, defined over $\mathbb{R}$, then $H_{1}(\mathbb{R})^{*}$ is weakly exponential. This can be seen as follows. Let $M$ be a Levi subgroup of $H_{1}$ which is defined over $\mathbb{R}$. Note that $H_{1}(\mathbb{R})^{*}=M(\mathbb{R})^{*} R_{u} H_{1}(\mathbb{R})$. As $L$ is a maximal reductive subgroup of $H$ defined over $\mathbb{R}$, there is a $g \in H(\mathbb{R})$, such that $g M g^{-1} \subset L$. Thus $g M(\mathbb{R}) g^{-1} \subset L(\mathbb{R})$. Thus $M(\mathbb{R})$ is a compact group. Recall that $R_{u} H_{1}(\mathbb{R})$ is a simply connected nilpotent Lie group. Thus both $M(\mathbb{R})^{*}$ and $R_{u} H_{1}(\mathbb{R})$ are exponential group. Now by, Corollary $2.1 \mathrm{~A}$, [Ho-M], the group $H(\mathbb{R})^{*}$ is weakly exponential.

Now, using Theorem 1.8 we conclude that $P_{n}: H(\mathbb{R}) \rightarrow H(\mathbb{R})$ is surjective if and only if $P_{n}: H(\mathbb{R})^{*} \rightarrow H(\mathbb{R})^{*}$ is surjective and $n$ is coprime to the order of the finite group $H(\mathbb{R}) / H(\mathbb{R})^{*}$. It follows from the above that for any unipotent element $u \in H(\mathbb{R})^{*}$ the group $Z_{H}(\mathbb{R})(u)^{*}$ is weakly exponential. Thus we apply Theorem 1.7 to see that $P_{n}: H(\mathbb{R})^{*} \rightarrow H(\mathbb{R})^{*}$ is surjective if and only if $n$ is coprime to the order of the finite groups $Z_{H(\mathbb{R})^{*}}(u) / Z_{H}(\mathbb{R})(u)^{*}$ for all unipotent elements $u \in H(\mathbb{R})^{*}$. Under isomorphism $\phi$, reinterpreting the above observations we get that, $P_{n}: G \rightarrow G$ is surjective if and only if $n$ is coprime to the orders of the finite groups $G / G^{*}$ and $Z_{G^{*}}(v) / Z_{G}(v)^{*}$ for all $v \in N$. Note that $K$ is a maximal compact group of $G$ and further, using Lemma 5.10, $Z_{K^{*}}(v)$ is a maximal compact subgroup of $Z_{G^{*}}(v)$. Appealing to Theorem 3.1, page 180-181, [H] we see that $G / G^{*}$ is isomorphic to $K / K^{*}$ and that, for all unipotent element $u \in N$, the group $Z_{G}(v) / Z_{G}(v)^{*}$ is isomorphic to $Z_{K^{*}}(v) / Z_{K}(v)^{*}$. This completes the proof.

Corollary 5.11. Let $H$ be a compact real Lie group, which is not necessarily connected and let $n$ be an integer. Then $P_{n}: H \rightarrow H$ is surjective if and only if $n$ is relatively prime to the order of $H / H^{*}$. Moreover, if $h \in H$ is such that the order of $h H^{*}$ in $H / H^{*}$ is relatively prime to $n$ then $h$ has a $n$-th root in $H$.

Proof. The first part follows immediately from Theorem 1.9. To prove the last part we consider the subgroup $H_{1}$ generated by $h$ and $H^{*}$, and then apply the first part of this corollary.

Remark 5.12. The above corollary generalises a classical theorem of Heinz Hopf which says that for a compact connected real Lie group all the $n$-th power maps are surjective (see [Hop]). The Corollary 5.11 also follows from Proposition 3.1 in [Br] due to R. Brown. The proof of Proposition 3.1 in $[\mathrm{Br}]$ depends on the cohomology theory of compact Lie groups, but our proof is very different and is a natural consequence of the results on the power maps of real algebraic groups proved in this paper. 


\section{ACKNOWLEDGMENTS}

The author thanks Dave Witte Morris for asking the question which led to this paper. The author also thanks Gopal Prasad for helpful discussions and comments.

\section{REFERENCES}

[B-M] A. Borel and G.D. Mostow, On semisimple automorphisms of Lie algebras, Ann. of Math., 61 : 389-405, 1955 .

[B-S] A. Borel and J.-P. Serre, Sur certains sous-groupes des groupes de Lie compacts, Comment. Math. Helv., 27 : 128-139, 1953.

[B-T] A. Borel and J. Tits, Groupes réductifs, Inst. Hautes Études Sci. Publ. Math., 27 : 55-150, 1965.

[Br] Robert F. Brown, On the power map in compact groups, Quart. J. Math. Oxford Ser. (2), 22 : 395-400, 1971.

[Ch1] Pralay Chatterjee, On the surjectivity of power maps of solvable Lie groups, J. Algebra, 248 : 669-687, 2002.

[Ch2] Pralay Chatterjee, On the surjectivity of the power maps of algebraic groups in characteristic zero, Math. Res. Letters, $9: 741-756,2002$.

[Ch3] Pralay Chatterjee, On the surjectivity of the power maps of semisimple algebraic groups, Math. Res. Letters, 10 : 625-633, 2003.

[Ch4] Pralay Chatterjee, On the power maps, orders and exponentiality of p-adic algebraic groups, J. Reine Angew. Math., 629 : 201-220, 2009.

[Ch5] Pralay Chatterjee, Surjectivity of power maps of real algebraic groups, preprint.

[Co-M] D.H. Collingwood and W.M. McGovern, Nilpotent orbits in semisimple Lie algebras. Van Nostrand Reinhold Co., New York, 1993.

[D-M] S.G. Dani and M. McCrudden, A criterion for exponentiality in certain Lie groups, J. Algebra, 238 : 82-98, 2001.

[Dj-Ho] D.Z. Djoković and K.H. Hofmann, The surjectivity questions for the exponential function of real Lie groups : a status report, J. Lie Theory, 7 : 171-197, 1997.

[Dj-T] D.Z. Djoković and N.Q. Thang, On the exponential map of almost simple real algebraic groups, J. Lie Theory, 5: 275-291, 1995.

[H] G. Hochschild, The structure of Lie groups, Holden-Day, Inc., 1965.

[Ho-M] K.H. Hofmann and A. Mukherjea, On the density of the image of the exponential function, Math. Ann., 234 : 263-273, 1978.

[Hop] Heinz Hopf, Über den Rang geschlossener Liescher Gruppen, Comment. Math. Helv. 13 : 119-143, 1940.

$[\mathrm{M}] \quad \mathrm{M}$. McCrudden, On $n$-th roots and infinitely divisible elements in a connected Lie group, Math. Proc. Cambridge Philos. Soc., 89: 293-299, 1981.

[P] Gopal Prasad, E-mail correspondence with the author, April, 2007.

[S] J.-P. Serre, Bounds for the orders of the finite subgroups of $G(k)$, Group representation theory, 405-450, EPFL Press, Lausanne, 2006.

[Sp] T.A. Springer Linear Algebraic Groups, Second Edition, PM-9, Birkhäuser, 1998.

[St] R. Steinberg, On the power maps in algebraic groups, Math. Res. Letters, 10: 621-624, 2003.

[St1] R. Steinberg, Endomorphisms of linear algebraic groups, Memoirs of the Amer. Math. Soc., 80: 1968.

[T-Y] P. Tauvel and R.W.T. Yu, Lie algebras and algebraic groups, Springer Monographs in Mathematics, Springer-Verlag, 2005.

The Institute of Mathematical Sciences, C.I.T. Campus, Taramani, Chennai- 600113, India $U R L:$ http://www.imsc.res.in/ ${ }^{\sim}$ pralay

E-mail address: pralay@imsc.res.in 